Revue internationale d'éducation de Sèvres

$24 \mid 1999$

Le droit à l'éducation : vers de nouveaux contenus pour le XXle siècle

\title{
Conférence introductive
}

\section{Claude Durand-Prinborgne}

\section{(2) OpenEdition \\ Journals}

Édition électronique

URL : http://journals.openedition.org/ries/2602

DOI : 10.4000/ries.2602

ISSN : 2261-4265

Éditeur

Centre international d'études pédagogiques

Édition imprimée

Date de publication : 1 décembre 1999

Pagination : 21-34

ISSN : $1254-4590$

\section{Référence électronique}

Claude Durand-Prinborgne, "Conférence introductive », Revue internationale d'éducation de Sèvres [En ligne], 24 | 1999, mis en ligne le 23 novembre 2012, consulté le 01 mai 2019. URL : http:// journals.openedition.org/ries/2602 ; DOI : 10.4000/ries.2602

\section{(c) Tous droits réservés}




\section{Conférence introductive}

Claude Durand-Prinborgne

Professeur émérite à l'université de Paris I

Ancien recteur d'académie 
La réflexion qu'il nous a été demandé de conduire en ouverture du colloque "Le droit à l'éducation : vers de nouveaux contenus pour le XxIe siècle ", est objectivement délicate pour plusieurs raisons. La notion même de " contenus » justifie trois observations.

En premier lieu, on notera que la littérature internationale des instances intervenant dans le secteur de l'éducation - l'UNESCO, la Banque mondiale, l'OCDE, le Conseil de l'Europe ou d'autres instances régionales - est relativement pauvre en ce qui concerne la notion même de contenus et l'approche de cette question, surtout si on la compare à tout ce qui est consacré au rôle des enseignants ', aux finalités ou aux objectifs de l'éducation, ou bien encore à ce qui est consacré aux concepts d'éducation de base, d'éducation fondamentale, d'éducation pour tous, d'éducation tout au long de la vie.

Tout semble se passer comme si les problèmes de contenus n'apparaissaient souvent qu'en situation de non-explicité, derrière l'affichage des objectifs, réduits en quelque sorte au rôle de conséquences que l'on pourrait logiquement déduire - et ceci est d'autant plus perceptible que l'on avance dans la réflexion sur la notion même de contenus.

La deuxième raison est que la question des «contenus »- et surtout " quels contenus pour le XXI siècle ? " - est un sujet vaste et sans limite précise. Il est en effet souvent difficile, quand ce n'est pas arbitraire, de séparer contenus, méthodes, enseignements, école, éducation formelle.

Il existe un troisième problème dès lors que l'on cherche à cerner ce sujet. Il apparaît directement tributaire de notions dont chacune relève de définitions en attente ou encore en discussion. Ainsi, dans ce colloque, on approche la question des contenus en la reliant à la notion d'éducation de base, en retrouvant la vieille opposition éducation formelle/éducation informelle, et en l'associant également à la notion d'éducation tout au long de la vie... Voilà ce qui constituerait un paquetage de départ conséquent, mais notre propos ne consiste pas à préciser toutes les notions en cause, parce que nous n'en aurions pas le temps, que ce serait trop ambitieux et que cela nous détournerait peut-être de notre but.

Quelques remarques, cependant. La première remarque, et elle n'est pas - il faut le préciser - critique, de quelque manière que ce soit, à l'égard des organisations, est qu'à lecture du titre (ou, plus exactement, des titres) du colloque, on relève une certaine ambiguïté. Le titre premier, « Le droit à l'éducation : vers de nouveaux contenus pour le XXI siècle » est clair. Mais ce titre est associé à une explicitation qui vient immédiatement après - explicitation qui n'en est pas véritablement une - en ces termes : «Comment l'éducation peut-elle répondre au

1 Par exemple, Le Rapport mondial sur l'éducation, UNESCO, 1998, sous le titre Les enseignants et l'enseignement dans un monde en mutation, ne comporte aucun développement sur les contenus. 
$\mathrm{XXI}^{\mathrm{e}}$ siècle aux défis planétaires du droit à l'éducation de base pour tous et de l'éducation de chacun tout au long de la vie, face à la mondialisation, à la modernisation et aux sociétés en mutation ?" Dans cette explicitation, certains renvois sont clairs. Le XXI ${ }^{\mathrm{e}}$ siècle circonscrit le temps. Le défi planétaire circonscrit l'espace à la mondialisation. La modernisation et la mutation circonscrivent la cause de la recherche de nouveaux contenus. Mais ensuite on se trouve renvoyé au droit à l'éducation, renvoyé à l'éducation de base, renvoyé à l'éducation tout au long de la vie.

Je ne reprendrai pas l'ensemble de ces notions sauf une : le droit à l'éducation. À partir du moment où l'on s'accorde pour reconnaître que, mis à part l'existence, et on ne peut que le regretter, de quelque 130 millions d'enfants qui n'ont pas accès à l'éducation et d'un nombre non négligeable d'adultes qui n'ont pas eu accès à l'éducation, le droit à l'éducation a tout de même fait quelques progrès, par rapport à la situation cinquante ans plus tôt, et les dispositions internationales, que je rappellerai tout à l'heure, sont des témoins d'une volonté politique passée. Les textes sont sans portée pour l'avenir sauf, et c'est là l'un des intérêts de fond du débat, si l'on dégage une nouvelle motivation à l'existence de ces textes, constituant une nouvelle base de départ pour l'affirmation d'un nouveau droit à l'éducation, ce qui passe effectivement avec de nouveaux contenus. L'analyse, sans doute assez juridique, est très réaliste dans l'ordre international et nous sommes là au centre même de la réflexion.

Arrivé à ce stade, il faut s'interroger sur ce que l'on entend par contenus, parce qu'il me semble que l'une des conditions sine qua non d'un travail fructueux, c'est d'être d'accord sinon sur la terminologie, du moins sur les concepts que nous manions, et de ne pas avoir l'attitude du politique qui peut se contenter de certaines imprécisions de termes. Je vois, pour ma part, trois notions mêmes de contenus lorsque l'on parle d'éducation. Première notion : le « contenu politique ", sans donner au terme une connotation péjorative. C'est la traduction du fait que tout système d'éducation national ou sub-national, tant pour l'éducation formelle que pour l'éducation informelle, consciemment ou inconsciemment (par un poids de l'histoire par exemple), assume de façon différente des autres systèmes des fonctions inhérentes à l'acte éducatif, qui sont des fonctions d'intégration sociale de l'individu, des fonctions de transmission des expériences vécues, de partition ou d'articulation entre tradition et innovation.

Il y a un deuxième sens, plus précis, que j’appellerai le « contenu-valeur », c'est-à-dire un contenu intentionnellement décidé par des politiques pour l'acte éducatif, donc un contenu voulu. On le retrouve dans l'ordre international comme dans les ordres nationaux, consistant par exemple à faire passer des messages touchant la responsabilité de l'individu, le travail, la probité, la démocratie, le respect des autres, les droits de l'homme, etc. Ceci pose les problèmes délicats de la relation des valeurs aux savoirs, aux disciplines ou aux apprentissages. On voit ici un éventail s'ouvrir. 
Enfin, il y a un troisième sens, encore plus précis, qu'on pourrait analyser comme étant le contenu utilisé par l'enseignant ou par celui qui a en charge une transmission dans la fonction d'éducation. Il s'agit alors du " contenu appliqué ", la déclinaison en somme des valeurs précédentes en termes de - et je multiplie la terminologie - matières, disciplines, programmes, modules, apprentissage. C'est le contenu concret de la transmission - savoirs, savoir-être, savoir-faire.

Ces trois sens ne sont pas en opposition, mais leur définition peut contribuer à aider à la réflexion.

Enfin, la question des contenus, il faut aussi le rappeler, va se situer dans le cadre d'un ordre mondial, défini politiquement et encadré juridiquement. Quand on parle ici d'ordre international, quelques périls nous guettent toujours. Il est nécessaire que nous soyons ambitieux pour donner les objectifs, pour favoriser l'évolution de l'humanité, pour répondre aux aspirations des générations futures. Mais en même temps il est nécessaire que nous n'ayons pas d'excès dans nos ambitions parce que nous risquerions de tomber dans un idéalisme rêveur ou de conduire à une certaine désespérance devant la non-réalisation. Nous devons être sans cesse confrontés dans nos propres choix à un critère de faisabilité et d'adéquation à des situations qui sont diverses.

L'ordre international peut proposer des orientations; les applications ne relèvent pas de l'ordre international. Elles relèvent des États, des nations ou de structures sub-nationales. Et nous ne pouvons pas oublier qu'il y a encore des États dans lesquels les enfants n'accèdent pas à l'éducation. Je souhaiterais que l'on pense ici à l'instituteur de brousse ou à l'enseignant de certains bidonvilles de grandes cités de pays en voie de développement, et que nous ayons une vision très concrète des choses.

Ceci posé, j'ai imaginé d'aborder le problème, c'est-à-dire l'instrumentalisation des options que peut faire l'UNESCO, en le prenant sous trois angles successifs qui me semblent pouvoir éclairer le débat. Même si cette terminologie n'est pas totalement satisfaisante, on peut distinguer une approche politique, une approche économico-sociale et une approche technicienne, que je qualifierai de "pédagogique ». Elles se traduisent toutes trois en choix de politiques éducatives.

\section{Approche politique}

L'expression s'entend dans deux sens : options politiques de la société internationale et options des politiques éducatives. La caractéristique de cette approche est d'être très souvent exprimée en termes de démarche finaliste. On fixe les finalités, on détermine des objectifs, on raisonne en termes de : "Je veux ceci donc je fais cela " ou " je demande que l'on fasse cela ». Qu'en est-il dans 
l'ordre international ? Quelles conséquences peut-on en tirer pour ce qui relève des contenus?

\section{Les dispositions internationales}

Il y a, initialement, un choix de société : celui de la communauté internationale, celui des Nations Unies. Ce choix de société se traduit à son tour en un choix fondamental de politique éducative instrumentalisé par l'UNESCO, conséquence de ce choix des Nations Unies qu'est la paix, avec un certain nombre de passages obligés : la tolérance, la compréhension des autres, la marche vers la démocratisation.

Je ne rappellerai pas l'article 26 de la Déclaration universelle des droits de l'homme sur le droit à l'éducation. On y trouve deux types de précisions. D'abord des dispositions relatives à l'organisation de l'éducation : ce qui touche à la gratuité, à l'obligation de l'enseignement élémentaire, à la généralisation de l'enseignement technique et professionnel, à l'accès aux études supérieures, aux droits des parents. Ensuite des indications de finalité : l'épanouissement de la personne humaine, le renforcement du respect des droits de l'homme, l'obligation de favoriser la compréhension, l'amitié entre les nations, les groupes raciaux ou religieux.

Le texte, certes, a cinquante ans. Mais il a été suivi d'un certain nombre d'autres textes qui ont progressivement procédé à ce que j'appellerai des « mises à jour ». Les questions que nous nous posons peuvent trouver des éléments de réponse, par exemple, dans l'article 28 de la Convention relative aux droits de l'enfant ${ }^{2}$, mais également dans la Convention internationale sur l'élimination de toute forme de discrimination raciale (janvier 1969) au titre du droit à l'éducation pour tous, dans le Pacte international relatif aux droits économiques, sociaux et culturels (janvier 1976), enfin dans la Conférence mondiale sur l'éducation pour tous (mars 1990, Jomtien). Elles en trouvent peut-être aussi, à côté des textes consacrés internationalement, dans les résultats des travaux de la Commission Delors sur l'éducation pour le XXI ${ }^{\text {e }}$ siècle.

\section{Les contenus}

Le passage de ce rappel de dispositions internationales à la réflexion sur les contenus entraîne quelques observations. Au fond, les dispositions internationales concernent principalement les contenus-valeurs. L’un des débats actuels porte sur la question de savoir si l'on n'est pas en train de passer, par une muta-

2 La Convention proclame le droit de tous les enfants à l'enseignement primaire qui leur donne les compétences nécessaires pour continuer à apprendre durant leur vie. Elle oriente vers un modèle d'enseignement centré sur l'enfant pour lui permettre de participer, de penser, d'apprendre à apprendre. 
tion de la notion de droit à l'éducation, des contenus-valeurs à la notion même de « contenus-matières".

Les dispositions internationales concernent donc les contenus-valeurs ; on peut imaginer qu'ensuite elles ont des conséquences sur les contenusmatières. Lorsque l'on parle de nouveaux contenus, cela ne concerne pas directement, semble-t-il, les contenus-valeurs parce que les valeurs internationales en cause, rappelées par ces dispositions échappent largement à la relativité dans le temps. La question est donc de savoir si nous ne donnons pas un nouveau sens aux valeurs, telles qu'elles ont été définies par les textes, ou si nous n'avons pas impulsé une nouvelle volonté de développer ces valeurs, de les porter dans la réalité du quotidien des différentes nations à un point plus extrême que celui qui serait atteint pour le moment.

Se pose alors une question clé : avons-nous franchi un pas - ce que je ne critiquerai pas personnellement - en passant d'une contrainte de l'international, consistant en une obligation de faire quelque chose, à savoir de réaliser la scolarisation, à une contrainte internationale dans laquelle on sentirait les premières affirmations d'une volonté de demander que l'on aille déjà plus loin. Nous n'en sommes plus à l'exigence de réaliser une scolarisation à $100 \%$, nous n'en sommes plus à l'exigence d'une scolarisation reposant sur un minimum traditionnel "lire-écrire-compter », nous en sommes à une exigence plus grande, parce que nous impliquons des valeurs dont nous pensons qu'elles ne sont pas encore totalement atteintes.

Sans insister davantage sur ce point, il me semble que si le siècle à venir débute par la célébration de l'Année internationale de la paix, les valeurs à transmettre par l'éducation sont et restent incontestablement la justice, la liberté, la paix dans le monde, les droits de l'homme, l'égale dignité de tous et la tolérance.

Or, comme nous savons tous que ces valeurs ne sont pas toujours respectées, qu'il y a des conflits localisés, que la situation des minorités dans certains cas est une situation extrêmement délicate, cela veut dire que la fonction d'éducation n'apporte encore pas assez d'instruments et de résultats au service des causes internationalement définies. Il faut donc de toute évidence continuer de réfléchir à la façon dont les contenus pourront faire passer ces messages en termes de valeurs. L'accord semble exister au plan des méthodes sur le point suivant : les contenus doivent être transmis à la fois par la voie d'un contenu-matière, mais aussi par des pratiques d'expérimentation et d'acquisition progressive.

$\mathrm{Au}$ fond, dans l'éducation de base, doivent se trouver un " enseignement " portant sur les valeurs qui viennent d'être évoquées et aussi une pratique. $\mathrm{Au}$ fur et à mesure que se développe le cursus scolaire, il doit y avoir une montée en puissance de la pratique de ces valeurs et non plus seulement de la réception de contenus -, qui s'épanouirait dans l'exercice par l'homme ou la femme de sa citoyenneté, à partir du moment où politiquement il/elle peut intervenir dans la gestion de la cité. 


\section{Approche économique et sociale}

On ne peut éluder cette approche, même si elle s'écarte des deux thèmes classiquement abordés : l'effet de l'éducation sur l'économie et la responsabilité de l'éducation dans le domaine de l'éducation au changement économique. Ce n'est pas cela qui est en cause.

Nous sommes confrontés d'abord à une mondialisation, ensuite à une modernisation, enfin à des sociétés en mutation. Ces évolutions ont des effets déterminants sur les systèmes éducatifs existant actuellement et sur les demandes qui leur sont adressées, telles que - pour simplifier - d'être des acteurs du changement. Deux observations s'imposent.

La première est que le libellé de certains documents, explicitement ou implicitement, est parfois gênant dans la mesure où l'approche des problèmes d'adaptation des systèmes éducatifs, des contenus, à un changement de société est exprimée en termes de déterminisme, c'est-à-dire comme adaptation nécessaire, imposée. En somme, on va partir de constats sur les structures de la société pour en tirer des conséquences en ce qui concerne nos systèmes éducatifs. Ce primat me paraît dominer de très nombreux raisonnements. Croire qu'il pourrait totalement en aller autrement relèverait de la naïveté ou de l'angélisme. Mais en même temps, il me plaît de rappeler ici, parce que c'est une conviction profonde, que les enjeux permanents de l'éducation, où que ce soit, ne sont pas réductibles uniquement à une adaptation à des techniques, à des finalités économiques, à des finalités sociales. Il existe d'autres enjeux en ce qui concerne l'éducation. Son rôle historique d'abord, son rôle présent et futur ne permettent pas d'enfermer l'éducation dans une fonction simplement technicienne de formation d'acteurs économiques ou d'êtres adaptés socialement. Elle est porteuse de valeurs. Elle doit former des hommes et des femmes dignes, libres, responsables.

La deuxième observation est qu'il existe, d'une part, un problème de hiérarchie entre les valeurs retenues par les dispositions internationales précédemment évoquées et, d'autre part, que cette préoccupation d'adaptation va, rapidement et largement, diffuser dans le monde un certain nombre de nouvelles valeurs susceptibles d'entrer en conflit avec des valeurs traditionnelles de certaines sociétés, mais également avec les valeurs internationales. Ceci justifie que l'on porte une attention vigilante à cette situation.

Un troisième point doit être rappelé ici : l'existence d'un risque de dissociation de la société internationale en deux groupes avec un écart croissant entre eux, comme nous le trouvons dans nos sociétés nationales, entre une minorité apte à évoluer dans un monde en mutation et une majorité qui subirait l'évolution de ce monde ou qui regarderait les choses se passer sans pouvoir s'impliquer. 


\section{Un monde en mutation}

On peut définir cette mutation par trois traits : un constat de mondialisation, un constat de globalisation et un développement de nouvelles technologies, qui est peut-être la cause principale des changements. Il y a en la matière une littérature suffisante pour que l'on n'y revienne pas. Nous ne sommes donc plus simplement dans l'interrogation d'un impact de changement, distant dans le temps, de caractère scientifique et technologique, sur l'éducation de base ; il s'agit maintenant de quelque chose de beaucoup plus profond. Si nous posons à ce moment la question en termes de mutations et de contenus, que peut-il se produire?

\section{Mutations et contenus}

Ces mutations sommairement résumées ont deux conséquences premières que je serais tenté de séparer ici : les nouvelles technologies d'une part, la mondialisation et la globalisation d'autre part.

\section{Les nouvelles technologies}

Que les nouvelles technologies aient un effet sur le cadre scolaire, cela va de soi. L'enfant, l'adolescent, l'adulte va trouver matière à connaissances, à apprentissages, à savoirs en dehors du cadre scolaire. Au sein même de celui-ci, la parole du maître, le savoir du maître, ne sont plus seuls, par exemple lors de la lecture d'un cédérom. Les connaissances stockées ne se réduisent plus au livre. La mémoire est même désormais dans les ordinateurs.

La conception d'une éducation tout au long de la vie s'en trouve modifiée. Il apparaît relativement aisé d'admettre que l'éducation de base peut simplement donner les instruments qui permettront d'accéder à l'éducation tout au long de la vie, d'apprendre à accéder à l'internet, par exemple. Les possibilités d'un enseignement à distance sont aussi modifiées.

S'agissant des nouvelles technologies - sauf à souligner des problèmes considérables de coût d'acquisition, sauf à dire que des questions d'éthique se posent -, si le cadre institutionnel de l'éducation de base peut être changé, il n'y a pour autant rien qui soit, si on le veut, véritablement infranchissable pour adapter les contenus aux nouvelles technologies.

Il peut y avoir un apprentissage généralisé de l'accès au clavier de l'ordinateur, on sait tous que cela ne pose pas de problème pour les enfants, mais beaucoup plus pour leurs parents et plus encore pour leurs grands-parents. Il peut $\mathrm{y}$ avoir une initiation plus ou moins poussée à d'autres aspects plus attachants intellectuellement de l'informatique. 
Il doit sans doute y avoir, et cela me paraît des plus importants, une formation à l'esprit critique, pour préparer l'élève, dès l'éducation de base, à faire le tri dans cette masse d'informations à laquelle il aura accès. Cette éducation à l'esprit critique peut être rapprochée des préoccupations touchant la mondialisation et des problèmes de globalisation.

\section{Globalisation et mondialisation}

Le cadre national, et non seulement l'école, risque d'être brisé, par l'internet, par les messageries électroniques. Toutes les oppositions classiques - dans l'école/hors école, dans le temps scolaire/hors temps scolaire, en formation initiale/en formation permanente, en éducation formelle/en éducation non formelle - sont affectées et perdent du sens. Les valeurs qui existent déjà sont transmises dans le cadre de la mondialisation, ce qui entraîne des conséquences sur les contenus-matières et le système éducatif.

Qu'attend-on du système éducatif ? Cette question est au cœur de nos préoccupations. On attend d'abord que l'éducation soit capable d'une adaptabilité permanente. Pas seulement qu'elle soit adaptée au temps T, mais qu'elle soit adaptable à l'évolution de la situation économique, technique et sociale.

On attend aussi que le système d'éducation joue un rôle d'information, un rôle de formation. La prise en charge de l'éducation par l'UNESCO va être confrontée au même type de problème que dans le domaine culturel. L'UNES$\mathrm{CO}$, au plan culturel, s'occupe - et c'est un succès considérable - de faire reconnaître une égale dignité des cultures et a réussi à empêcher que certaines d'entre elles ne disparaissent, ne soient gommées. Il n'est pas certain que les choses soient aussi avancées sur le plan de l'éducation, qu'il n'existe pas une tendance à définir un modèle dominant de type occidental, européen, nord-américain. Et pourtant on ne peut que souhaiter que le passé de certains des systèmes éducatifs soit considéré comme un aspect du patrimoine mondial de l'humanité et classé comme peut l'être un monument.

L'un des grands problèmes des responsables politiques actuels est d'admettre la mutation - tout en permettant la poursuite d'un développement harmonieux des cultures diverses, telles que leur histoire les a forgées, de garder cette richesse considérable.

Reste alors ce qui parait le plus difficile. Dans la recherche de nouveaux contenus, nous trouvons, quel que soit le sens que l'on donne au terme, des attentes bien définies, soit d'ordre collectif ou individuel, soit relatives aux qualités nécessaires pour demain (la créativité, la responsabilité, l'adaptation de l'être humain, la possibilité d'un travail avec d'autres...). Mais si tout cela est facile à dire, il est beaucoup plus difficile de déterminer si l'on transmet des réponses à ces attentes et à ces nécessités, et comment. Avec quels outils ? Avec quel savoir ? Avec quel concours disciplinaire ? Comment faire apprendre le goût 
du travail en équipe ? Comment faire apprendre l'esprit d'innovation ? Comment faire apprendre la créativité ? Comment faire apprendre le sens de la réalité et des réalités? Comment le concilier avec l'épanouissement individuel ?

Ce qui frappe pourtant est que tout ceci est autant nécessaire à l'adaptation des contenus face aux mutations économiques, sociales et techniques, qu'à la poursuite de l'action en faveur des valeurs (démocratie, droits de l'homme, respect des convictions...).

Les deux phénomènes de mondialisation et de globalisation posent une vraie question évoquée par des philosophes, des politiques et des sociologues : l'élargissement de l'espace accessible au plus grand nombre - aidé par les techniques - conduit-il aussi vers l'universel ou, en réaction, à une montée de replis identitaires ? ${ }^{3}$ L'éducation est elle-même au cœur du problème puisqu'elle est à la fois porteuse des promesses d'avenir de l'Universel et gardienne des richesses des passés culturels.

Ces questions sont les plus délicates et les plus essentielles pour faire prévaloir dans l'éducation de base une véritable formation au lieu d'une simple instruction et pour que se développe ensuite une éducation tout au long de la vie.

\section{Approche pédagogique}

La terminologie ne satisfait pas totalement. Elle est réductrice. Elle n'est qu'un pis-aller. C'est une approche technique qui pourrait être conduite, pour partie par des politiques, pour partie, plus largement, par des techniciens des sciences de l'éducation et des acteurs responsables de l'éducation. La nature de leur intervention paraît simple à préciser. Les finalités politiques de l'éducation pour l'homme et pour la société internationale sont définies par l'exigence d'adaptation au monde en mutation formulée dans son principe et dans ses conséquences. Il s'agit donc de rechercher et de déterminer les modes opératoires les plus efficaces pour atteindre les finalités et réaliser les objectifs. Mais il est important de savoir comment. Là où la Commission Jacques Delors sur l'éducation pour le XXI ${ }^{\text {e }}$ siècle dit " apprendre à être ", l'écho est "comment le fait-on?", " apprendre à apprendre? " : comment ?

Des deux approches précédemment retenues, nous avons pu sans doute déjà tirer des propositions. L'approche " politique ", au-delà de la permanence des valeurs à transmettre, à inculquer, suppose - et c'est là sans doute que réside la nouveauté - qu'une attention plus grande soit portée au rôle de l'éducation dans les domaines des droits de l'homme, des libertés politiques, de la démocra-

3 Cf. travaux de Samuel Huntington, Dominique Wolton, Jean-Marie Domenach, Edgar Morin, Benjamin Barber. 
tie politique et sociale, etc. L'approche " économico-sociale " permet aussi de déduire des conséquences, même si cela est beaucoup moins facile. Cette double finalité conduit à des exigences communes.

Dans un cas comme dans l'autre, l'exigence première est de favoriser la capacité de penser d'une façon critique - ce qui passe par l'apprentissage actif, la création, le débat, l'échange. Ceci sera tout autant nécessaire au citoyen responsable qu'à l'homme ou la femme dans le monde nouveau.

Dans la poursuite de la finalité d'adaptation aux mutations du monde économique et technique et dans la poursuite de finalités de paix mondiale, de respect de l'autre, d'appartenance à la communauté des hommes, il faut développer les attitudes civiques, la coopération, la solidarité.

Doit-on, par une acception large, inclure dans les contenus certains des aspects du fonctionnement de l'institution scolaire ? Si on l'admet, on peut considérer comme de plus en plus essentiel qu'à côté de l'enseignement, de l'éducation civique, de "l'information-formation " sur les libertés, sur l'égalité des races, des sexes, sur le droit de participer, les modes de fonctionnement incluent des modes effectifs de participation. Au-delà de l'intérêt de la démarche pour une éducation à la démocratie, ceci, a-t-on pu constater, réduit les attitudes de passivité scolaire.

L'approche "pédagogique " constitue un terrain propre de réflexion autour des notions de savoirs, d'apprentissage, de techniques. Cette interrogation cerne le possible. Elle détermine un " comment ", qui n'est pas seulement fait de la simple exécution d'une partition écrite par d'autres, les décideurs. Le système se ferme en boucle : chaque interrogation ici met en cause le sens et l'intérêt des options politiques, économiques et sociales et en dégage les effets.

\section{Les savoirs}

Ce qui caractérise le monde actuel - c'est une banalité de le redire - et sans doute le monde futur, c'est, d'abord, le double mouvement qui affecte le savoir, un prodigieux développement et une compartimentation, et, ensuite, la rapidité des acquisitions et de l'évolution.

L'éducation ne doit être le lieu de transmission ni d'un savoir spécialisé ignorant le global, ni d'un savoir global qui ne comprenne rien au local. Au premier titre, celui du constat de l'opposition développement/compartimentation, on retrouve, sous une autre forme, l'opposition global/local, universel/partiel.

$\mathrm{Au}$ second titre, deux constats s'imposent. Le premier, le plus récent, le plus original aussi, est exprimé par Michel Serres dans un résumé sur le résultat d'une évolution : "Toute l'histoire le démontre, à chaque changement de support a correspondu une transformation du savoir ". Il évoque le passage de l'oral à l'écrit, de l'écrit à l'imprimé ; le savoir se trouve aujourd'hui dans les mémoires d'ordinateur, sur les écrans et les réseaux. L'idée capitale est que le chemin d'ac- 
cès au savoir s'inverse : les hommes mobiles se déplaçaient vers le savoir, le savoir diffusé se déplace désormais vers les hommes. Le problème consiste à détenir la capacité technique d'accès.

Le second constat, plus ancien déjà, reste d'actualité et permanent : c'est l'accélération du rythme des transformations, c'est le passage de la génération à la petite dizaine d'années et l'expansion du savoir. Les conséquences de cette situation ne sont pas les mêmes selon les niveaux de l'enseignement. Pour l'enseignement de base, le constat justifie deux démarches : la première vise à trier dans les savoirs ce qui doit être retenu, la seconde consiste à créer l'articulation entre des spécialistes disciplinaires ou à construire l'enseignant généraliste.

Si l'on admet que l'éducation a en charge de procurer trois types de savoirs, les savoirs de base (lire, écrire, compter), les savoir-faire et les savoir-être, il est facile de mesurer les différences d'enjeux dans la prise en compte des finalités politiques et socio-économiques. Pour les savoirs de base, l'évolution prévisible met en cause principalement la question des modes de transmission, notamment techniques, et éventuellement, mais il faut encore rester modeste dans l'ordre international, celle de l'élévation du niveau d'exigence. Ce savoir de base doit être une fondation mais aussi une sorte de bagage minimum, la valise de tout homme ; il doit être conçu pour élever des étages sur des fondations solides.

Le problème est tout autre pour les savoir-faire. Il s'agit du développement de nouvelles aptitudes. C'est là que se posent toutes les questions relatives aux disciplines, aux relations entre elles, au choix des contenus, aux évaluations. Il y a là un très vaste chantier de révision des choix concrets des politiques éducatives.

Le problème enfin, en ce qui concerne les savoir-être, est celui du développement de nouvelles attitudes comportementales : aptitude au travail en équipe, mobilité, polyvalence, esprit d'initiative. Il s'agit moins de savoirs que de compétences.

\section{Les techniques}

Il n'est pas utile de souligner l'importance de la mutation de la société, avec le développement des ordinateurs, des robots, de l'intelligence artificielle, mais on ne peut faire l'économie d'une triple réflexion. Son premier volet, déjà précédemment évoqué, est celui de l'énorme risque d'écarts entre les nations quant à l'accès et à l'appropriation des nouvelles techniques, pour des raisons économiques de coûts des équipements. Le second est moins international, il reste propre à chacune des sociétés, mais il est d'autant plus grave que la relation entre les générations reste plus ou moins fortement marquée par la considération portée à l'âge. Les pays riches connaissent bien déjà l'opposition entre les 
facilités d'accès des jeunes générations aux nouvelles techniques et le refus d'approche ou la peur de certaines personnes âgées. Comment éviter des conflits de générations dans d'autres sociétés ? Il ne s'agit plus seulement ici, en effet, de donner à des jeunes l'instrument qu'est la lecture, mais bien d'autre chose !

Le troisième volet est celui de la nature du rôle assigné aux nouvelles technologies de l'information et de la communication, situé entre deux extrêmes théoriquement concevables : l'asservissement total à l'éducation ou l'affranchissement comportant le risque de dépérissement, de disparition de l'éducation.

C'est dans cette perspective qu'il faut souligner au moins, dénoncer sans doute, ce qu'ont favorisé souvent et favorisent peut-être encore les aides techniques bilatérales. Il s'agit de la transposition pure et simple d'arguments ou de méthodes, validés en pays développés, dans des pays en voie de développement. On a cité, par exemple, le développement de la pré-scolarisation là où la scolarisation est de $25 \%$, la réduction des effectifs des classes, l'amélioration de la qualité des manuels. Ceci peut tout aussi bien concerner les contenus, comme par exemple l'incitation à la théorisation.

\section{Apprentissage}

Prendre le mot à part, le séparer des savoirs, a pour mérite principal de déplacer le problème de l'éducation pour le $\mathrm{XXI}^{\mathrm{e}}$ siècle, en passant de la question "quelles connaissances? » à la question "quelle formation?».

Si l'on admet le concept d'éducation tout au long de la vie, les conséquences en termes de contenus sont capitales, que l'on entende contenus au sens de valeurs ou au sens de programmes. Les contenus-valeurs inculqués dans l'éducation de base à l'enfant seront entretenus. Les contenus-programmes ne se bouclent pas sur eux-mêmes dans une éducation de base en fournissant seulement des instruments d'épanouissement et de progrès à chacun; ils sont le socle, pour une construction qui se poursuit, de l'ensemble des savoirs et de l'accès à ceux-ci. En même temps, l'éducation tout au long de la vie permet de trouver une réponse aux problèmes de l'adaptation à la mobilité du monde, au changement rapide.

Mais, et ceci sera "le mot de la fin " pour introduire le trouble nécessaire à l'interrogation, la question posée est centrée sur les contenus... Or l'on sait que deux conceptions s'affrontent en éducation : celle de la démarche et celle des contenus. Il est permis de s'interroger sur la pertinence qu'il peut y avoir à paraitre retenir les seuls contenus. Pour apprendre, apprendre à faire, et sans doute, pour nombre de situations éducatives à faire évoluer et nombre d'objectifs à poursuivre et à atteindre, il serait peut-être plus efficace de mettre l'accent sur les démarches. 